\section{FRI0272 SAFETY PROFILE IN PATIENTS WITH PSORIASIS, PSORIATIC ARTHRITIS AND ANKYLOSING SPONDYLITIS OVER LONG TERM: UPDATED POOLED SAFETY ANALYSES}

A. Deodhar ${ }^{1}$, I. Mcinnes ${ }^{2}$, X. Baraliakos ${ }^{3}$, K. Reich ${ }^{4,5}$, A. B. Gottlieb ${ }^{6}$, M. Lebwohl ${ }^{7}$, S. Schreiber ${ }^{8}$, W. Bao ${ }^{9}$, K. Marfo ${ }^{10}$, H. Richards ${ }^{10}$, L. Pricop ${ }^{9}$, A. Shete ${ }^{10}$, J. Safi ${ }^{9}$, P. J. Mease ${ }^{11} .{ }^{1}$ Oregon Health \& Science University, Portland, United States of America; ${ }^{2}$ University of Glasgow, Glasgow, United Kingdom; ${ }^{3}$ Ruhr-University Bochum, Bochum, Germany; ${ }^{4}$ University Medical Center Hamburg-Eppendorf, Hamburg, Germany; ${ }^{5}$ Skinflammation ${ }^{\oplus}$ Center, Hamburg, Germany; ${ }^{6}$ Icahn School of Medicine at Mount Sinai, New York, United States of America; ${ }^{7}$ Mount Sinai Hospital, New York, United States of America; ${ }^{8}$ University Hospital Schleswig-Holstein, Kiel, Germany; ${ }^{9}$ Novartis Pharmaceuticals Corp., East Hanover, United States of America; ${ }^{10}$ Novartis Pharma AG, Basel, Switzerland; ${ }^{11}$ Swedish Medical Center and University of Washington, Seattle, United States of America

Background: Pooled safety data has been reported with secukinumab (SEC) in patients (pts) with Psoriatic arthritis (PsA), Ankylosing Spondylitis (AS) and Psoriasis (PsO). ${ }^{1}$

Objectives: To report longer-term safety data of SEC treatment in PsA, AS, PsO pts up to 5 years.

Methods: The integrated clinical trial safety dataset included data pooled from 28 randomised controlled clinical trials of SEC 300 or 150 or $75 \mathrm{mg}$ in PsO (11 Phase 3 and 8 Phase 4 trials), PsA (5 Phase 3 trials), and AS (4 Phase 3 trials), along with post-marketing safety surveillance data with a cut-off date of 25 December 2018. Adverse events (AEs) were reported as exposure-adjusted incident rates (EAIRs) per 100 pt-years. Analyses included all pts who received $\geq 1$ dose of SEC.

Results: A total of 12637 pts $(8819,2678$ and 1140 pts with PsO, PsA and AS, with an exposure of 15063.1, 5984.6 and 3527.2 pt-years, respectively) were included. The most frequent $A E$ was upper respiratory tract infection and EAIR per 100 pt-years for IBD, malignancies and MACE remained low. The EAIR per 100 pt-years for adverse events (AEs) of special interest are reported in Table 1. The cumulative post-marketing exposure to SEC was estimated to be $\sim 285,811$ pt-years across the approved indications. Safety data from post-marketing surveillance are reported in Table 2.

Table 1. Selected AEs of interest with SEC across pooled clinical trials

\begin{tabular}{lccc}
\hline Variable & PsO & PsA & AS \\
\cline { 2 - 4 } & SEC & SEC & SEC \\
& $\mathbf{N}=\mathbf{8 8 1 9}$ & $\mathbf{N = 2 6 7 8}$ & $\mathbf{N = 1 1 4 0}$ \\
\hline Exposure (Days), Mean (SD) & $623.9(567.7)$ & $816.2(580.7)$ & $1130.1(583.0)$ \\
Death, $\mathrm{n}(\%)$ & $15(0.2)$ & $13(0.5)$ & $10(0.9)$ \\
Selected AE's of interest, EAIR (95\% Cl) & & \\
Serious infections $^{1}$ & $1.4(1.2,1.6)$ & $1.8(1.5,2.2)$ & $1.2(0.9,1.6)$ \\
Candida infections $^{2}$ & $2.9(2.7,3.2)$ & $1.5(1.2,1.9)$ & $0.7(0.5,1.1)$ \\
IBD $^{3}$ & $0.01(0.0,0.05)$ & $0.03(0.0,0.1)$ & $0.03(0.0,0.2)$ \\
Crohn's disease $^{3}$ & $0.1(0.05,0.2)$ & $0.1(0.04,0.2)$ & $0.4(0.24,0.7)$ \\
Ulcerative colitis $^{3}$ & $0.1(0.08,0.2)$ & $0.1(0.04,0.2)$ & $0.2(0.1,0.5)$ \\
MACE $^{4}$ & $0.4(0.31,0.5)$ & $0.4(0.3,0.6)$ & $0.7(0.4,1.0)$ \\
Uveitis $^{3}$ & $0.01(0.0,0.05)$ & $0.1(0.04,0.2)$ & $1.2(0.9,1.7)$ \\
Malignancy $^{5}$ & $0.9(0.7,1.0)$ & $1.0(0.77,1.3)$ & $0.5(0.3,0.8)$ \\
\end{tabular}

${ }^{1}$ Rates for system organ class; ${ }^{2}$ Rates for high level term; ${ }^{3}$ Rates for preferred term (PT: IBD for unspecified IBD); ${ }^{4}$ Rates for Novartis MedDRA Query term; ${ }^{5}$ Rates for standardized MedDRA query term - 'malignancies and unspecified tumour'; EAIR, exposure adjusted incidence rate per 100 pt-years; $\mathrm{N}$, number of pts in the analysis

Table 2. Summary of SEC post-marketing safety

\begin{tabular}{|c|c|c|c|c|c|c|c|}
\hline \multirow[t]{2}{*}{ Exposure (PTY) } & $\begin{array}{c}\text { PSUR1 } \\
\text { 26Dec14 } \\
\text {-25Jun15 }\end{array}$ & $\begin{array}{c}\text { PSUR2 } \\
\text { 26 Jun } \\
-25 \text { Dec15 }\end{array}$ & $\begin{array}{c}\text { PSUR3 } \\
\text { 26Dec15 } \\
-25 J u n 16\end{array}$ & $\begin{array}{l}\text { PSUR4 } \\
\text { 26Jun } \\
\text {-25Dec16 }\end{array}$ & $\begin{array}{c}\text { PSUR5 } \\
\text { 26Dec16 } \\
-25 \text { Dec17 }\end{array}$ & $\begin{array}{c}\text { PSUR6 } \\
\text { 26Dec17 } \\
\text {-25Dec18 }\end{array}$ & \multirow{2}{*}{$\begin{array}{l}\text { Cumulative } \\
285811\end{array}$} \\
\hline & 1838 & 7450 & 16871 & 28549 & 93744 & 137325 & \\
\hline & \multicolumn{7}{|c|}{ n (Reporting rate PTY) } \\
\hline $\begin{array}{l}\text { Serious } \\
\text { infections }\end{array}$ & $89(4.8)$ & $149(2.0)$ & $232(1.4)$ & $475(1.7)$ & $649(0.7)$ & $1841(1.3)$ & $3980(1.4)$ \\
\hline Malignancy & $2(0.1)$ & $15(0.2)$ & $21(0.1)$ & $50(0.2)$ & $225(0.2)$ & $422(0.3)$ & $788(0.3)$ \\
\hline Total IBD & $4(0.2)$ & $12(0.2)$ & $37(0.2)$ & $46(0.2)$ & $185(0.2)$ & $340(0.3)$ & $693(0.2)$ \\
\hline MACE & $6(0.3)$ & $15(0.2)$ & $16(0.1)$ & $39(0.1)$ & $151(0.2)$ & $238(0.2)$ & $504(0.2)$ \\
\hline
\end{tabular}

PSUR, periodic safety update report; PTY, pt-treatment years

Conclusion: In this long-term analysis across clinical trials and post-marketing surveillance, of pts with $\mathrm{PsO}, \mathrm{PsA}$ and AS, SEC was well tolerated, with a safety profile consistent with previous reports. ${ }^{1}$

\section{Reference:}

[1] Deodhar et al. Arthritis Research \& Therapy (2019) 21:111

Disclosure of Interests: Atul Deodhar Grant/research support from: AbbVie, Eli Lilly, GSK, Novartis, Pfizer, UCB, Consultant of: AbbVie, Amgen, Boehringer Ingelheim, Bristol Myer Squibb (BMS), Eli Lilly, GSK, Janssen, Novartis, Pfizer UCB, Speakers bureau: AbbVie, Amgen, Boehringer Ingelheim, Bristol Myer Squibb (BMS), Eli Lilly, GSK, Janssen, Novartis, Pfizer, UCB, lain Mclnnes Grant/research support from: Bristol-Myers Squibb, Celgene, Eli Lilly and Company, Janssen, and UCB, Consultant of: AbbVie, Bristol-Myers Squibb, Celgene, Eli Lilly and Company, Gilead, Janssen, Novartis, Pfizer, and UCB, Xenofon Baraliakos Grant/research support from: Grant/research support from: AbbVie, BMS, Celgene, Chugai, Merck, Novartis, Pfizer, UCB and Werfen, Consultan of: AbbVie, BMS, Celgene, Chugai, Merck, Novartis, Pfizer, UCB and Werfen, Speakers bureau: AbbVie, BMS, Celgene, Chugai, Merck, Novartis, Pfizer, UCB and Werfen, Kristian Reich Grant/research support from: Affibody; Almirall; Amgen; Biogen; Boehringer Ingelheim; Celgene; Centocor; Covagen; Eli Lilly; Forward Pharma; Fresenius Medical Care; GlaxoSmithKline; Janssen; Kyowa Kirin; LEO Pharma; Medac; Merck; Novartis; Miltenyi Biotec; Ocean Pharma; Pfizer; Regeneron; Samsung Bioepis; Sanofi Genzyme; Takeda; UCB; Valeant and Xenoport., Consultant of: Affibody; Almirall; Amgen; Biogen; Boehringer Ingelheim; Celgene; Centocor; Covagen; Eli Lilly; Forward Pharma; Fresenius Medical Care; GlaxoSmithKline; Janssen; Kyowa Kirin; LEO Pharma; Medac; Merck; Novartis; Miltenyi Biotec; Ocean Pharma; Pfizer; Regeneron; Samsung Bioepis; Sanofi Genzyme; Takeda; UCB; Valeant and Xenoport., Speakers bureau: Affibody; Almirall; Amgen; Biogen; Boehringer Ingelheim; Celgene; Centocor; Covagen; Eli Lilly; Forward Pharma; Fresenius Medical Care; GlaxoSmithKline; Janssen; Kyowa Kirin; LEO Pharma; Medac; Merck; Novartis Miltenyi Biotec; Ocean Pharma; Pfizer; Regeneron; Samsung Bioepis; Sanofi Genzyme; Takeda; UCB; Valeant and Xenoport., Alice B Gottlieb Grant/research support from:: Research grants, consultation fees, or speaker honoraria for lectures from: Pfizer, AbbVie, BMS, Lilly, MSD, Novartis, Roche, Sanofi, Sandoz, Nordic, Celltrion and UCB., Consultant of:: Research grants, consultation fees, or speaker honoraria for lectures from: Pfizer, AbbVie, BMS, Lilly, MSD, Novartis, Roche, Sanofi, Sandoz, Nordic, Celltrion and UCB., Speakers bureau: Research grants, consultation fees, or speaker honoraria for lectures from: Pfizer, AbbVie, BMS, Lilly, MSD, Novartis, Roche, Sanofi, Sandoz, Nordic, Celltrion and UCB., Mark Lebwohl Grant/research support from: AbbVie, Amgen, Arcutis, AstraZeneca, Boehringer Ingelheim, Celgene, Clinuvel, Eli Lilly, Incyte, Janssen Research \& Development, LLC, Kadmon Corp., LLC, Leo Pharmaceutucals, Medimmune, Novartis, Ortho Dermatologics, Pfizer, Sciderm, UCB, Inc. and ViDac, Consultant of: Allergan, Almirall, Arcutis, Inc., Avotres Therapeutics, BirchBioMed Inc., Boehringer-Ingelheim, Bristol-Myers Squibb, Cara Therapeutics, Castle Biosciences, Corrona, Dermavant Sciences, Evelo, Foundation for Research and Education in Dermatology, Inozyme Pharma, LEO Pharma, Meiji Seika Pharma, Menlo, Mitsubishi, Neuroderm, Pfizer, Promius/Dr. Reddy's Laboratories, Theravance, and Verrica, Stefan Schreiber Consultant of: AbbVie, Arena, BMS, Biogen, Celltrion, Celgene, IMAB, Gilead, MSD, Mylan, Pfizer, Fresenius, Janssen, Takeda, Theravance, provention Bio, Protagonist and Falk, Weibin Bao Shareholder of: Novartis, Employee of: Novartis, Kwaku Marfo Shareholder of: Novartis, Employee of: Novartis, Hanno Richards Shareholder of: Novartis, Employee of: Novartis, Luminita Pricop Shareholder of: Novartis Employee of: Novartis, Abhijit Shete Shareholder of: Novartis, Employee of: Novartis, Jorge Safi Shareholder of: Novartis, Employee of: Novartis, Philip $J$ Mease Grant/research support from: Abbott, Amgen, Biogen Idec, BMS, Celgene Corporation, Eli Lilly, Novartis, Pfizer, Sun Pharmaceutical, UCB - grant/ research support, Consultant of: Abbott, Amgen, Biogen Idec, BMS, Celgene Corporation, Eli Lilly, Novartis, Pfizer, Sun Pharmaceutical, UCB - consultant, Speakers bureau: Abbott, Amgen, Biogen Idec, BMS, Eli Lilly, Genentech, Janssen, Pfizer, UCB - speakers bureau DOI: 10.1136/annrheumdis-2020-eular.5118

\section{\begin{tabular}{|l|l|l|l}
\hline FRI0273 EFFECTIVENESS AND RETENTION RATE OF \\
\hline
\end{tabular} SECUKINUMAB FOR PSORIATIC ARTHRITIS AND AXIAL SPONDYLOARTHRITIS: REAL-LIFE DATA FROM THE ITALIAN LORHEN REGISTRY}

E. G. Favallii ${ }^{1}$ A. Marchesoni ${ }^{1}$, S. Balduzzi ${ }^{2}$, C. Montecucco ${ }^{3}$, C. Lomater ${ }^{4}$ G. Crepaldi ${ }^{4}$, S. Talamini ${ }^{5}$, C. Bazzani ${ }^{5}$, E. Fusaro ${ }^{6}$, M. Priora ${ }^{6}$, A. lannello ${ }^{7}$, G. Paolazzi ${ }^{8}$, R. Caporali ${ }^{1,9} .{ }^{1}$ Gaetano Pini-CTO Institute, Department of Rheumatology, Milan, Italy; ' 2 Università di Pavia, IRCCS Policlinico San Matteo, Rheumatology, Pavia, Italy; ${ }^{3}$ Università di Pavia, IRCCS Policlinico San Matteo, Pavia, Italy; ${ }^{4} \mathrm{AO}$ Ordine Mauriziano, SSD Reumatologia, Torino, Italy; ${ }^{5}$ Spedali Civili, UO Reumatologia e Immunologia Clinica, Brescia, Italy; ${ }^{6}$ A.O.U. Città della Salute e della Scienza di Torino, SC Reumatologia, Torino, Italy; ${ }^{7}$ Day Hospital Multidisciplinare PO SS. Trinità, Borgomanero, Italy; ${ }^{8}$ Ospedale Santa Chiara, UOC di Reumatologia, Trento, Italy; ${ }^{9}$ Università degli Studi di Milano, Department of Clinical Sciences \& Community Health, Milano, Italy 